\title{
Fisioterapia respiratória associada à pressão positiva nas vias aéreas na evolução pós-operatória da cirurgia bariátrica
}

\author{
Respiratory physiotherapy associated with airway positive pressure in the \\ postoperative bariatric surgery evolution
}

Fabiana Sobral Peixoto-Souza', Bruna Gallo-Silva', Luciana Bernardo Echevarria², Marcio Antonio Antunes Silva ${ }^{2}$, Elisane Pessoti ${ }^{3}$, Eli Maria Pazzianotto-Forti ${ }^{4}$

\begin{abstract}
I To assess the tidal volume (VT), minute volume (MV) and respiratory rate (RR) of morbidly obese women in postoperative bariatric surgery (BS), after the conventional respiratory physiotherapy (CRP) with or without preoperatively continuous positive airway pressure (CPAP). Thirty-six women, aged $40.1 \pm 8.41$ years, that would be submitted to BS by laparotomy were studied. All of them underwent preoperative outpatient conventional respiratory physiotherapy (CRP) (diaphragmatic breathing exercises, deep breathing, fractionated breathing and breathing associated to movements of the upper limbs, a series of 10 repetitions of each exercise) by 30 days. Once admitted, 18 were subjected to 20 minutes of CPAP, an hour before induction of anesthesia and composed group CRP+CPAP. The other 18 did not receive CPAP and composed group CRP. VT, $\mathrm{MV}$, and RR were performed through the ventilometer, at admission and 24 hours after surgery. It was found that measures of TV, VM and RR were not statistically significant when comparing pre and postoperative in both groups and as well as when the two groups were compared between them in both pre and postoperative. The results suggest that both the application and the implementation of FRC and FRC+CPAP in the preoperative period contribute to the maintenance of respiratory variables in the postoperative period. The use of CPAP before induction of anesthesia, did not provide additional benefits in the post-bariatric surgery with regard to the volumes.
\end{abstract}

Keywords I bariatric surgery; continuous positive airway pressure; lung volume measurements; physical therapy specialty.
RESUMOI Analisar volume corrente (VC), volume minuto (VM) e frequência respiratória (FR) de obesas mórbidas no pós-operatório de cirurgia bariátrica (CB), após a fisioterapia respiratória convencional (FRC) associada ou não à pressão positiva contínua nas vias aéreas (CPAP) no pré-operatório. Foram estudadas 36 mulheres, com idade de 40,1 8 ,41 anos, que seriam submetidas à CB por laparotomia e que realizaram FRC (exercícios respiratórios diafragmáticos, de inspirações profundas, fracionadas e associados a movimentos de membros superiores, 1 série de 10 repetições de cada exercício) por 30 dias antes da cirurgia. Após internação, 18 delas foram submetidas a 20 minutos de CPAP, 1 hora antes da indução anestésica e compuseram o grupo FRC+CPAP. As outras 18 não receberam o CPAP e compuseram o grupo FRC. Foram avaliados VM, VC e FR por meio do ventilômetro, no momento da internação e 24 horas após a realização da cirurgia. Constatou-se que as medidas de VC, VMe FR não apresentaram significância estatística quando comparados os resultados do pré e pós-operatório em ambos os grupos, bem como quando comparados os dois grupos entre si tanto no pré como no pós-operatório. Os resultados sugerem que a tanto a aplicação da FRC como a aplicação da FRC+CPAP no período pré-operatório contribui para a manutenção das variáveis respiratórias no pós-operatório. A aplicação do CPAP antes da indução anestésica não promoveu benefícios adicionais no pós-operatório de CB no que se refere aos volumes pulmonares. Descritores I cirurgia bariátrica; pressão positiva contínua nas vias aéreas; medidas de volume pulmonar; fisioterapia.

Estudo desenvolvido no curso de Fisioterapia, no Programa de Mestrado em Fisioterapia da Faculdade de Ciências da Saúde da Universidade Metodista de Piracicaba (UNIMEP); Clínica Bariátrica, Centro de Gastroenterologia e Cirurgia de Obesidade do Hospital dos Fornecedores de Cana de Piracicaba - Piracicaba (SP), Brasil.

'Fisioterapeuta; Programa de Mestrado em Fisioterapia da UNIMEP - Piracicaba (SP), Brasil.

2Fisioterapeuta; curso de Graduação em Fisioterapia da UNIMEP - Piracicaba (SP), Brasil.

3Mestre em Fisioterapia pela UNIMEP; Fisioterapeuta da Clínica Bariátrica de Piracicaba - Piracicaba (SP), Brasil.

${ }^{4}$ Fisioterapeuta; Professora Doutora, docente do curso de Graduação em Fisioterapia e do Programa de Pós-graduação em Fisioterapia da UNIMEP - Piracicaba (SP), Brasil. 


\section{INTRODUÇÃO}

A obesidade é uma doença crônica que pode alcançar proporções alarmantes prejudicando a saúde física, psicossocial e o bem-estar ${ }^{1}$. A intervenção mais eficaz na condução clínica dos obesos de grandes proporções ou mórbidos é a cirurgia bariátrica ${ }^{2,3}$.

Entretanto, os procedimentos cirúrgicos abdominais altos, incluindo as gastroplastias ${ }^{4}$, afetam a musculatura respiratória por meio de diferentes mecanismos, tais como a perda da integridade muscular pela incisão cirúrgica, uso de bloqueadores neuromusculares durante a anestesia ou outros mecanismos indiretos como a dor e a incisão ${ }^{5}$, favorecendo dessa forma a alteração muscular respiratória determinando diminuição dos volumes e capacidades pulmonares e aumento da frequência respiratória ${ }^{4}$.

Além da diminuição dos volumes e capacidades pulmonares, decorrentes da cirurgia, ainda podem ocorrer complicações pulmonares pós-operatórias (CPP), dentre elas a pneumonia, as atelectasias e o broncoespasmo ${ }^{6-11}$.

Também existem evidências de que a disfunção diafragmática seja um fator relevante na etiologia das CPP. Este fato parece advir da manipulação das vísceras durante o procedimento cirúrgico, gerando inibição reflexa do nervo frênico e, consequentemente, a paresia temporária do músculo diafragma ${ }^{12}$, embasando a necessidade da fisioterapia respiratória nesses pacientes ${ }^{13}$.

A prevenção das complicações pós-operatórias pulmonares é um dos objetivos mais importantes do tratamento de pacientes submetidos à cirurgias ${ }^{14}$. Segundo Tzani, Chetta e Olivieri ${ }^{14}$, as intervenções preventivas, incluindo o controle da dor, fisioterapia respiratória e pressão positiva contínua nas vias, pode efetivamente reduzir a ocorrência de complicações pulmonares. Os principais objetivos da fisioterapia respiratória incluem promover a reexpansão pulmonar, restaurar volumes e capacidades pulmonares, facilitar a expectoração de secreções traqueobrônquicas e melhorar a condição de imobilidade funcional acarretada pelo repouso no leito, minimizando assim as complicações que facilmente acometem pacientes obesos e cirúrgicos ${ }^{6,15-20}$.

Considerando que a aplicação de pressão positiva contínua nas vias aéreas (CPAP) apresenta efeito benéfico para resolução das complicações pulmonares ${ }^{14}$ por restaurar volumes e capacidades pulmonares, aumentar a oxigenação e melhorar a mecânica muscular respiratória ${ }^{15}$, a hipótese do presente estudo foi que a utilização da pressão positiva associada à fisioterapia respiratória convencional durante o período pré-operatório poderia trazer benefícios adicionais à restauração do volume corrente
(VC) da frequência respiratória (FR) e do volume minuto $(\mathrm{VM})$ quase sempre alterados dentro das primeiras 24 horas do pós-operatório de cirurgias bariátricas.

Assim, o objetivo do presente estudo foi analisar o comportamento do VC, do VM e da FR no pós-operatório de cirurgia bariátrica, após a aplicação de fisioterapia respiratória convencional (FRC) associada ou não à pressão positiva contínua nas vias aéreas (CPAP), no pré-operatório de cirurgia bariátrica.

\section{METODOLOGIA}

Este estudo foi aprovado pelo Comitê de Ética em Pesquisa sob o Parecer n ${ }^{\circ}$ 30/09 e desenvolvido seguindo as orientações da Resolução no 196/96 do Conselho Nacional de Saúde.

O cálculo amostral foi realizado por meio do software BioEstat ${ }^{\circledR}$, versão 5.0, sendo estabelecido poder estatístico de $80 \%$ e $\alpha$ de 0.05 . A variável desfecho considerada para o cálculo amostral foi volume corrente. Baseando-se em estudos prévios ${ }^{13}$, foi estabelecido o número de 18 voluntárias em cada grupo.

Trata-se de estudo intervencionista e transversal no qual foram estudadas 36 mulheres, com diagnóstico de obesidade mórbida segundo classificação da OMS (IMC $>40 \mathrm{~kg} / \mathrm{m}^{2}$ ), não tabagistas e/ou portadores de doenças pulmonares agudas ou crônicas, que se encontravam internadas e seriam submetidas à cirurgia bariátrica pela técnica do bypass gástrico em Y de Roux, por laparotomia. Todas as voluntárias estudadas participaram de programa de fisioterapia pré-operatória ambulatorial, no qual receberam orientações sobre a cirurgia sendo salientada a importância da realização de exercícios respiratórios para a prevenção das complicações pulmonares no pós-operatório. O programa teve início 30 dias antes da cirurgia; as voluntárias foram orientadas sobre os exercícios respiratórios de inspirações profundas e fracionadas, o uso do incentivador respiratório, o treino da tosse com contensão abdominal e os exercícios metabólicos de extremidades. As voluntárias foram orientadas para a continuidade dos exercícios em casa e a realizarem uma série de dez exercícios para cada atividade proposta, uma vez por dia, durante todos os dias da semana até no dia da internação hospitalar. O programa foi conduzido por fisioterapeuta respiratório e os encontros com as voluntárias foram realizados uma vez por semana.

No momento da internação, as voluntárias foram divididas, por sorteio, em 2 grupos iguais com 18 voluntárias 
cada. $\mathrm{O}$ primeiro denominado grupo $\mathrm{FRC}+\mathrm{CPAP}$ e o segundo grupo FRC. As voluntárias foram informadas e esclarecidas a respeito dos objetivos e da metodologia experimental aos quais seriam submetidas, sendo explicitado o caráter não invasivo dos procedimentos.

$\mathrm{Na}$ fase pré-operatória, após a internação hospitalar, as voluntárias foram avaliadas quanto aos dados pessoais, antropométricos, os sinais vitais (FR, FC e PA) e os volumes pulmonares.

Para a realização das medidas dos volumes pulmonares foi utilizado um ventilômetro OHMEDA Respirometer $^{\circledR}$ (modelo RM121, Japão) demarcado em litros. As medidas do VM foram realizadas acoplando-se o ventilômetro a uma máscara facial sendo que as voluntárias permaneceram na posição sentada e foram orientadas a respirar normalmente por um minuto conectadas ao ventilômetro. Por meio de um cronômetro, foi possível avaliar a FR durante o procedimento e o VC foi calculado de forma indireta, dividindo-se o $\mathrm{VM}$ pela $\mathrm{FR}^{21}$. $\mathrm{O}$ procedimento foi realizado 3 vezes ou mais sendo padronizado intervalo de 3 minutos entre eles até que foram obtidos 3 valores próximos entre si, ou seja, com diferença menor que $10 \%$ entre eles. A medida de maior valor foi considerada para a análise.

No dia da cirurgia, uma hora antes da indução anestésica, no grupo $\mathrm{FRC}+\mathrm{CPAP}$, foi realizada a aplicação de CPAP, por 20 minutos estando as voluntárias no leito hospitalar, na posição semi-sentada, ou seja, com a cabeceira da cama elevada a $45^{\circ}$.

Para a realização do CPAP foi utilizado um gerador de fluxo Caradyne ${ }^{\circledast}$ (Ireland; WhisperFlow) ligado a uma rede de oxigênio por uma mangueira condutora de $\mathrm{O}_{2}$ e uma válvula reguladora de pressão. Por meio de uma traqueia corrugada, o gerador de fluxo foi conectado a máscara facial siliconizada, com válvula unidirecional sendo que no ramo expiratório da máscara foi colocada uma válvula do tipo Spring loaded (Vital Signs, EUA), a qual ofereceu ao sistema uma pressão positiva expiratória final (PEEP) de $10 \mathrm{cmH}_{2} \mathrm{O}$. A máscara foi acoplada à face das voluntárias através de fixadores de borracha. $\mathrm{O}$ grupo FRC não realizou exercícios no dia da cirurgia.

No primeiro dia do pós-operatório, ou seja, 24 horas após a cirurgia, as medidas do VM, VC e FR foram novamente realizadas. Em seguida, os dois grupos receberam FRC, duas vezes por dia, até a alta hospitalar.

A FRC consistiu em exercícios respiratórios diafragmáticos, exercícios de inspirações profundas, inspirações fracionadas em dois e três tempos e exercícios respiratórios associados ao movimento de flexão do ombro com extensão dos membros superiores. Para cada exercício proposto, foi realizada uma série de dez repetições. Foram realizados também exercícios para a prevenção da trombose venosa profunda e deambulação ${ }^{13}$.

Não houve perda amostral, todas as voluntárias foram submetidas ao mesmo procedimento cirúrgico, derivação gástrica em Y de Roux, mesmo procedimento anestésico, tempo cirúrgico semelhantes, permaneceram o mesmo tempo internadas (três dias) e não apresentaram complicações no pós-operatório, durante o período de internação.

\section{Análise estatística}

A análise estatística dos dados foi aplicada a partir do teste de normalidade de Shapiro-Wilk e, depois de rejeitada a hipótese de normalidade, aplicou-se o teste de significância dos dados a partir do teste não paramétrico de Wilcoxon (comparação intragrupo) e o teste de Mann-Withney (comparação entre grupos). Ambos com nível de significância de 5\% ( $\mathrm{p}<0,05)$.

\section{RESULTADOS}

Foram incluídos no estudo 36 pacientes do sexo feminino; sendo 18 voluntárias pertencentes ao grupo FRC+CPAP e 18 do grupo FRC.

A idade e características antropométricas das voluntárias estão dispostas na Tabela 1.

Quando avaliados os resultados da análise estatística referentes às medidas ventilométricas de VC, VM e FR pôde-se constatar que não houve diferença estatisticamente significativa quando comparados os resultados do pré e pós-operatório no grupo $\mathrm{FRC}+\mathrm{CPAP}$, assim como no grupo FRC. Da mesma forma, não foram significativos os resultados estatísticos na avaliação entre os grupos $\mathrm{FRC}+\mathrm{CPAP}$ e FRC tanto no pré quanto no pós-operatório, conforme mostra a Tabela 2.

Tabela 1. Idade e características antropométricas das voluntárias estudadas que compuseram os grupos fisioterapia respiratória convencional e fisioterapia respiratória convencional associada à pressão positiva contínua nas vias aéreas, no pré e pós-operatório de cirurgia bariátrica

$\begin{array}{lcc}\text { Grupos } & \text { FRC+CPAP }(n=18) & \text { FRC }(n=18) \\ \text { Idade (anos) } & 35,93 \pm 6,8 & 36,8 \pm 8,35^{*} \\ \text { Estatura }(\mathrm{m}) & 1,61 \pm 0,05 & 1,62 \pm 0,06^{*} \\ \text { Massa corporal }(\mathrm{kg}) & 117,28 \pm 9,98 & 114,7 \pm 9,18^{*} \\ \text { IMC }\left(\mathrm{kg} / \mathrm{m}^{2}\right) & 44,99 \pm 3,92 & 44,00 \pm 2,7^{*}\end{array}$

FRC: grupo fisioterapia respiratória convencional; FRC+CPAP: grupos fisioterapia respiratória convencional associada à pressão positiva contínua nas vias aéreas; IMC: índice de massa corpórea. *diferenç̧a não significativa 


\section{DISCUSSÃO}

Neste estudo, constatou-se que as medidas de VC, VM e FR não apresentaram significância estatística quando comparados os resultados do pré e pós-operatório tanto no grupo FRC, como no grupo $\mathrm{FRC}+\mathrm{CPAP}$, bem como quando comparadas os dois grupos entre si tanto no pré como no pós-operatório. Esse estudo foi realizado com o compromisso de contribuir na investigação em relação aos recursos fisioterapêuticos utilizados nos pós-operatórios e seus consideráveis benefícios, uma vez que a literatura aponta redução do $\mathrm{VC}$ e aumento da FR neste período e os resultados revelaram manutenção do VC, da FR e assim do VM após a aplicação dos tratamentos propostos.

$\mathrm{Craig}^{22}$, Ali et al. ${ }^{23} \mathrm{e}$ Dureuil, Cantineau e Desmonts ${ }^{24}$ afirmaram que a cirurgia bariátrica interfere diretamente na função pulmonar e que no pós-operatório propiciam o aparecimento de um padrão do tipo restritivo. Observaram a redução do $\mathrm{VC}$, da capacidade vital (CV), da força muscular respiratória e da pressão arterial de oxigênio. $\mathrm{O}$ aumento da frequência FR ocorre como necessidade de manutenção do VM dentro da normalida$\mathrm{de}^{11}$. Isso pode ser explicado pelo fato de que a anestesia promove o aumento da diferença do gradiente alvéolo-arterial de oxigênio o qual deve ser compensado com o aumento da ventilação na tentativa de se manter uma adequada oxigenação arterial. Esta situação se prolonga no $\mathrm{PO}$ por tempo variado, podendo deteriorar a função pulmonar, porém parece estar mais acentuada dentro das primeiras 24 horas $^{25}$.

Segundo Pelosi et al. ${ }^{26}$ a diminuição dos volumes pulmonares, sugere a presença de significante colapso pulmonar nos pacientes obesos além da propensão a desenvolver complicações pulmonares no pós-operatório acarretando em decréscimo da oxigenação.

Além disso, $\mathrm{Sue}^{27}$ relata que o conteúdo abdominal de indivíduos obesos favorece a compressão do abdome e do tórax restringido sua mobilidade.
No presente estudo não foram encontradas diferenças entre os volumes pulmonares quando comparados antes da realização da cirurgia e no primeiro dia do pós-operatório. Da mesma forma, quando comparadas às medidas do VC, VM e FR entre os dois grupos, tanto no pré como no pós-operatório, os resultados não evidenciaram diferenças significativas. Isso sugere que as propostas terapêuticas empregadas no pré-operatório podem ter sido efetivas na manutenção dos volumes e da frequência respiratória no período pós-operatório.

A aplicação da FRC pode ter contribuído para tanto; uma vez Costa et al. ${ }^{13}$, avaliando pacientes obesas mórbidas no pós-operatório da cirurgia bariátrica; também constataram resultados semelhantes após programa de FRC, ou seja, houve a manutenção dos volumes e capacidades pulmonares.

A aplicação do CPAP apresenta vários efeitos benéficos como aumento da pressão média das vias aéreas, recrutamento de alvéolos mal ventilados, aumento dos volumes pulmonares e estabilização das vias aéreas superiores ${ }^{28}$.

Por outro lado, de acordo com Squadrone et al. ${ }^{29}$, a utilização do CPAP pode ser subutilizada no período pré-operatório no tratamento de pacientes obesos, para recrutamento de áreas pulmonares com atelectasias. Isso se deve pelo aumento de tecido adiposo na região da orofaringe e cervical dificultando a pressurização das vias aéreas. Talvez esse tenha sido o motivo pelo qual não foi encontrado incremento do volume corrente no pós-operatório de cirurgia bariátrica, neste estudo. Entretanto, enfatizamos a importância tanto da FRC como da FRC+CPAP na manutenção dos volumes e FR no pós-operatório de cirurgia bariátrica.

Outro motivo para que o grupo $\mathrm{FRC}+\mathrm{CPAP}$ não tenha evidenciado um maior ganho de volume corrente em relação ao grupo FRC pode ter sido em função da oscilação da pressão positiva gerada no equipamento que varia de acordo com a combinação do fluxo gerado pela máquina e da válvula expiratória

Tabela 2. Médias e desvios padrão das medidas de frequência respiratória, volume corrente e volume minuto nos grupos fisioterapia respiratória convencional e fisioterapia respiratória convencional associada à pressão positiva contínua nas vias aéreas, no pré e pós-operatório de cirurgia bariátrica

\begin{tabular}{|c|c|c|c|}
\hline & & Pré-operatório & Primeiro dia pós-operatório \\
\hline \multirow{2}{*}{ Frequência respiratória (rpm) } & $\mathrm{FRC}+\mathrm{CPAP}$ & $15 \pm 1,8$ & $17,1 \pm 3,15^{*}$ \\
\hline & FRC & $15 \pm 5$ & $19,5 \pm 5^{\star}$ \\
\hline \multirow{2}{*}{ Volume corrente $(\mathrm{mL})$} & $\mathrm{FRC}+\mathrm{CPAP}$ & $574 \pm 84$ & $553 \pm 99 *$ \\
\hline & FRC & $600 \pm 114$ & $510 \pm 133^{*}$ \\
\hline \multirow{2}{*}{ Volume minuto (L) } & $\mathrm{FRC}+\mathrm{CPAP}$ & $8,2 \pm 2,0$ & $9,4 \pm 2,4^{*}$ \\
\hline & FRC & $8,8 \pm 2,3$ & $10,1 \pm 2,3^{*}$ \\
\hline
\end{tabular}

FRC: grupo fisioterapia respiratória convencional; FRC+CPAP: grupos fisioterapia respiratória convencional associada à pressão positiva contínua nas vias aéreas. *diferença estatistica não significativa 
utilizada $^{30}$, que pode ter de certa forma, subotimizado o recurso em questão.

Por outro lado, a manutenção do VC, VM e FR no primeiro dia do pós-operatório, ou seja, nas primeiras 24 horas após a cirurgia, para as quais se tem relato da presença da disfunção diafragmática, acarretando na diminuição dos volumes pulmonares e capacidades pulmonares $^{23,25}$, pode ser atribuído à realização da FRC pré-operatória ambulatorial.

Sabe-se que a disfunção diafragmática está presente em maior ou menor grau nas cirurgias abdominais, isto se deve por motivos inerentes ao ato cirúrgico como manipulação das vísceras no intraoperatório, a dor, a perda da integridade muscular abdominal o uso de bloqueadores musculares ${ }^{5}$, entre outros, justificando a intervenção fisioterapêutica neste pacientes ${ }^{13}$. Sendo assim esses resultados contribuem com a fisioterapia respiratória, especialmente na investigação de recursos a serem utilizados em pacientes cirúrgicos, pois segundo Lawrence et al. ${ }^{31}$, sabe-se que ao realizar a fisioterapia respiratória em pacientes em pré e pós-operatórios de cirurgias abdominais é melhor do que não realizar, entretanto, não se tem evidência de quais são os recursos mais indicados.

Considerando que pacientes obesos mórbidos especialmente os idosos ou portadores de síndrome da hipoventilação e apneia obstrutiva do sono são os que apresentam maior risco no desenvolvimento de complicações respiratórias ${ }^{32}$, estratégias que contribuam para a preservação da função pulmonar no pós-operatório são de relevância para a fisioterapia.

Pode ser considerado fator limitante do estudo a ausência da realização de teste espirométrico, por meio do qual poderiam ser exploradas outras variáveis ventilatórias importantes no seguimento respiratório de pacientes obesos mórbidos.

\section{CONCLUSÃO}

Os resultados sugerem que a tanto a aplicação da FRC como a aplicação da FRC+CPAP no período pré-operatório contribuem para a manutenção das variáveis respiratórias nas primeiras 24 horas do pós-operatório. A aplicação do CPAP, por 20 minutos, antes da indução anestésica, não promoveu benefícios adicionais no pós-operatório de cirurgia bariátrica no que se refere ao incremento dos volumes pulmonares, entretanto, contribuiu para a preservação deles.

\section{REFERÊNCIAS}

1. Formigueira X, Cantón A. Obesity: epidemiology and clinical aspects. Best Pract Res Clin Gastroenterol. 2004:18(6):1125-46.

2. Segal A, Fandino J. Indicações e contra-indicações para a realização das operações bariátricas. Rev Bras Psiquiatr. 2002;24(Suppl 3):68-72.

3. Consenso Bariátrico Brasileiro [cited 2012 Apr 08]. Available from: www.sbcb.org.br/imagens/pdf/consenso_baraitrico_brasileiro.pdf

4. Barbalho-Moulin MC, Miguel, GPS, Forti, EMP, Campos FA, Costa D. Effects of preoperative inspiratory muscle training in obese women undergoing open bariatric surgery: respiratory muscle strength, lung volumes and diaphragmatic excursion. Clinics (Sao Paulo). 2011;66(10):1721-7.

5. Siafakas NM, Mitrouska I, Bouros D, Georgopoulos D. Surgery and the respiratory muscles. Thorax. 1999;54(5):458-65.

6. Saad IAB, Zambom L. Variáveis clínicas de risco pré-operatório. Rev Assoc Med Bras. 2001;47(2):117-24.

7. Bagatini A, Trindade RD, Gomes CB. Anestesia para a cirurgia bariátrica: avaliação retrospectiva e revisão da literatura. Rev Bras Anestesiol. 2006;56(3):205-22.

8. Csendes A, Maluenda F. Morbimortalidad de la cirurgía bariátrica: experiência chilena em 10 instituciones de salud. Rev Chil Cir. 2006;58(3):208-12.

9. Faresin SM, Filardo FA, Fernandes ALG. Validade de um índice prognóstico para ocorrência de complicações pulmonares no pós-operatório de cirurgia abadominal alta. Rev Assoc Med Bras. 2002;48(3):209-16.

10. Silva EF, Guedes RP, Ribeiro EC. Estudo das repercussões das cirurgias abdominais sobre os músculos respiratórios. Fisioter Mov. 2003:16(1):51-6.

11. Eichenberger A, Proietti S, Wicky S, Frascarolo P, Suter M, Sapan DR, et al. Morbid obesity and postoperative pulmonary atelectasis: an underestimated problem. Anesth Analg. 2002;95(6):1788-92.

12. Ramos GC, Pereira E, Gabriel-Neto S, Oliveira EC. Aspectos históricos da pressão arterial de oxigênio e espirometria relacionados à operação abdominal. Arq Bras Cir Dig 2009;22(1):50-6.

13. Costa D, Forti EMP, Barbalho-Moulim MC, Rasera-Junior I. Estudo dos volumes pulmonares e da mobilidade toracoabdominal de portadoras de obesidade mórbida, submetidas à cirurgia bariátrica, tratadas com duas diferentes técnicas de fisioterapia. Rev Bras Fisioter. 2009;13(4):294-300.

14. Tzani P, Chetta A, Olivieri D. Patient assessment and prevention of pulmonary side-effects in surgery. Curr Opin Anaesthesiol. 2011:24(1):2-7.

15. Cardoso FPF, Osella OFS. Manovacuometria e ventilometria de mulheres obesas no pré-operatório de gastroplastia redutora [dissertação]. Brasília, DF: Universidade Católica de Brasília; 2005.

16. Forti EMP, Ike D, Barbalho-Moulim, MC, Rasera Junior I, Costa D. Effects of chest physioterapy on the respiratory function of postoperative gastroplasty patients. Clinics (Sao Paulo). 2009;64(7):683-9.

17. Polin RA, Sahni R. Newer experience with CPAP. Semin Neonatol. 2002;7(5):379-389

18. Fagevik Olsén M, Hahn I, Nordgren S, Lönroth H, Lundholm K. Randomized controlled trial of prophylactic chest physiotherapy in major abdominal surgery. Br J Surg. 1997;84(11):1535-8.

19. Souza RV, Denari SC, Ruiz SAL, Baroni,R, Montagnini AL, et al. Complicações pulmonares após cirurgias abdominais altas. O Papel da fisioterapia respiratória: revisão deliteratura. Rev ActaOncologicaBras.2002:22(4):348-54. 
20. El-solh A, Sikka P, Bozkanat E, Jaafar W, Davies J. Morbid obesity in the medical ICU. Chest. 2001;120(6):1989-97.

21. Gregorini C, Cipriano Junior G, Aquino LM, Branco JNR, Bernardelli GF. Estimulação elétrica nervosa transcutânea de curta duração no pósoperatório de cirurgia cardíaca. Arq Bras Cardiol. 2010;94(3):345-51.

22. Craig DB. Postoperative recovery of pulmonary function. Anesth Analg. 1981;60(1):46-52.

23. Ali J, Weisel RD, Layug AB, Kripke BJ, Hechtman HB. Consequences of postoperative alterations in respiratory mechanics. Am J Surg. 1974;128(3):376-82

24. Dureuil B, Cantineau JP, Desmonts JM. Effects of upper or lower abdominal surgery on diaphragmatic function. Br J Anaesth. 1987;59(10):1230-5.

25. Melero A, Vallés J, Vila P, Canet J, Vidal F. Recuperación anestésica, intercambio gaseoso y función hepática y renal postoperatorios en pacientes con obesidad mórbida sometidos a cirugía bariátrica: comparación de los efectos del halotano, isoflurano y fentanil. Rev Esp Anestesiol Reanim. 1993:40(5):268-72.

26. Pelosi P, Croci M, Ravagnan I, Vicardi P, Gattinoni L. Total respiratory system, lung, and chest wall mechanics in sedated-paralyzed postoperative morbidly obese patients. Chest. 1996;109(1):144-51.
27. Sue DY. Obesity and pulmonary function: more or less? Chest. 1997:111(4):844-5.

28. Crummy F, Naughton T. Non-invasive positive pressure ventilation for acute respiratory failure: justified or just hot air? Intern Med J. 2007;37(2):112-8

29. Squadrone V, Coha M, Cerutti E, Schellino MM, Biolino P, Occella $P$, et al. Continuous positive airway pressure for treatment of postoperative hypoxemia: a randomized controlled Trial. JAMA. 2005;293(5):589-95

30. Glover GW, Fletcher SJ. Assessing the performance of the Wisperflow continuous positive airway pressure generator: a bench Study. $\mathrm{Br} J$ Anaesth. 2009;102(6):875-81.

31. Lawrence VA, Cornell JE, Smetana GW, American College of Physicians. Strategies to reduce postoperative pulmonary complications after noncardiothoracic surgery: systematic review for the american college of physicians. Clinical Guideline. Ann Intern Med. 2006;144(8):596 - 608

32. Delgado PM, Lunardi AC. Complicações respiratórias pósoperatórias em cirurgia bariátrica: revisão da literatura. Fisioter Pesq. 2011;18(4):388-92. 